\title{
Macrophages are related to goblet cell hyperplasia and induce MUC5B but not MUC5AC in human bronchus epithelial cells
}

\author{
Manuel A Silva and Premysl Bercik
}

Airway goblet cell hyperplasia (GCH) — detectable by mucin staining—and abnormal macrophage infiltrate are pathological features present in many chronic respiratory disorders. However, it is unknown if both factors are associated. Using in-vivo and in-vitro models, we investigated whether macrophages are related with GCH and changes in mucin immunophenotypes. Lung sections from Sprague-Dawley rats treated for $48 \mathrm{~h}$ with one intra-tracheal dose of PBS or LPS ( $n=4-6$ per group) were immunophenotyped for rat-goblet cells, immune, and proliferation markers. Human monocytederived macrophages (MDM) were pre-treated with or without LPS, immunophenotyped, and their supernatant, as well as cytokines at levels equivalent to supernatant were used to challenge primary culture of normal human bronchus epithelial cells (HBEC) in air-liquid interface, followed by MUC5B and MUC5AC mucin immunostaining. An association between increased bronchiolar goblet cells and terminal-bronchiolar proliferative epithelial cells confirmed the presence of GCH in our LPS rat model, which was related with augmented bronchiolar CD68 macrophage infiltration. The in-vitro experiments have shown that MUC5AC phenotype was inhibited when HBEC were challenged with supernatant from MDM pre-treated with or without LPS. In contrast, TNF- $\alpha$ and interleukin- $1 \beta$ at levels equivalent to supernatant from LPS-treated MDM increased MUC5AC. MUC5B was induced by LPS, supernatant from LPS-treated MDM, a mix of cytokines including TNF- $\alpha$ and TNF- $\alpha$ alone at levels present in supernatant from LPS-treated MDM. We demonstrated that macrophages are related with bronchiolar GCH, and that they induced MUC5B and inhibited MUC5AC in HBEC, suggesting a role for them in the pathogenesis of airway MUC5B-related GCH.

Laboratory Investigation (2012) 92, 937-948; doi:10.1038/labinvest.2012.15; published online 5 March 2012

KEYWORDS: airways; cytokines; LPS; lung inflammation; TNF-alpha

Mucus is a gel that covers the airway epithelium and is constituted by different glycoproteins called mucins, which are secreted by goblet cells from submucosal glands and the airway epithelium. ${ }^{1}$ Mucus dissolves noxious gases and entraps foreign particles and pathogens, facilitating their clearance by mucociliary transport as a mechanism of innate defense. ${ }^{2}$ However, in chronic inflammatory respiratory diseases, such as asthma, chronic obstructive pulmonary disease (COPD), and cystic fibrosis (CF), there is a pathological goblet cell hyperplasia $(\mathrm{GCH})$ and mucus hypersecretion that can generate airway occlusion, associated with morbidity and death. ${ }^{1}$

The main gel-forming mucins found in human airways are MUC5B and MUC5AC. ${ }^{1}$ In healthy individuals, low levels of MUC5B and MUC5AC can be detected in mucus; although MUC5B is mainly present in the submucosal glands and
MUC5AC is predominantly distributed in the airway epithelium. ${ }^{3}$ In baseline conditions of chronic inflammatory respiratory disorders, MUC5B and/or MUC5AC are increased in different compartments of the respiratory tract. ${ }^{3-6}$ For example, MUC5B and MUC5AC are augmented in small airway lumen and epithelium from patients with advanced $\mathrm{CF} ;{ }^{4} \mathrm{GCH}$ related only with increased MUC5AC phenotype has been reported in bronchus from mild and moderate asthmatic patients; ${ }^{5}$ and abnormally high amounts of MUC5B and MUC5AC are present in the sputum from individuals with COPD, MUC5B being the predominant mucin. ${ }^{6}$ MUC5B and MUC5AC can be upregulated when human bronchus epithelial cells (HBEC) are cultured with retinoic acid, interleukin (IL)-6, IL-17, and neuroregulin$1 \beta 1 .^{7-11}$ MUC5AC can also be induced in normal HBEC by 
IL-13, ${ }^{12-14}$ although inhibited by thyroid hormone and epidermal growth factor. ${ }^{8}$ Furthermore, $\mathrm{MUC}^{2} \mathrm{AC}^{+} \mathrm{GCH}$ induced by IL-13 in normal HBEC depends on activation of IL-13R-JAK-STAT6 and EGFR-MAPK signaling pathways, as well as NF- $\kappa \mathrm{B},{ }^{15}$ relevant in allergic asthma as IL-13 is related to the pathogenesis of this disease. ${ }^{16}$ The regulation of MUC5B is less known, but studies using HBEC from smokers and healthy controls showed upregulation induced by oxidative stress from cigarette smoke and reactive oxygen species, ${ }^{3,17}$ relevant agents in the etiology of COPD.

Macrophages are resident cells originated from migrating peripheral blood monocytes (PBMC) that are present in all types of tissues. ${ }^{18,19}$ They maintain homeostasis when performing remodeling and wound healing, and can also execute an effector or regulatory immune response. ${ }^{18}$ Macrophages in normal rodents are found in distinctive alveolar and interstitial pulmonary compartments ${ }^{20,21}$ where they have different functional properties. ${ }^{22,23}$ In humans, lung macrophages are increased in the $\mathrm{CF}^{24}$ as well as in adult-but not pediatric - patients with severe eosinophilic asthma, ${ }^{25,26}$ and in individuals with severe COPD. ${ }^{27}$ Their role in asthma is largely unknown, ${ }^{28}$ but an impairment of phagocytic and antimicrobial functions in CF and COPD suggest that lung macrophages may have a key role in these two pathologies. ${ }^{29,30}$ Interstitial lung macrophages are difficult to obtain, and isolation of alveolar macrophages from bronchoalveolar lavage requires an invasive clinical procedure that limits their access for research. Functionally equivalent macrophages generated from PBMC differentiated in vitro with hematopoietic growth factors ${ }^{19,31,32}$ provide an alternative approach to study these cells.

In our knowledge, macrophages have never been related to the pathogenesis of airway GCH. In the present study, an anatomical association between GCH and macrophage infiltration was found in bronchioles from a rat model of lung inflammation induced by LPS when comparing phenotypes of large and small airways. These observations suggest that mediators released by the LPS-stimulated macrophages may induce changes in mucin phenotype of the airway goblet cells. This hypothesis was tested in vitro by using primary normal HBEC cultured in air-liquid interface and exposing them basolaterally to supernatant from human monocyte-derived macrophages (MDM) and cytokines at levels in those supernatant. Our results show that MDM supernatant and some related cytokines change the MUC5AC and MUC5B immunophenotypes, suggesting that macrophages could be involved in the pathogenesis of GCH.

\section{MATERIALS AND METHODS}

\section{Reagents for Immunostaining}

Antibody $(\mathrm{Ab})$-diluting buffer and Mayer's hematoxylin were obtained from Dako. Goat IgG and mouse $\operatorname{IgG}_{1}$ isotype controls were purchased from Southern Biotech. Customprepared monoclonal mouse anti-human MUC5B (clone
C12/3FB) from Novartis (Horsham, West Sussex, UK) was generated and validated as previously reported. ${ }^{10}$

\section{Animal Model}

Male Sprague-Dwaley rats (300-350 g) received intra-tracheal administration of PBS (control) or LPS $300 \mu \mathrm{g} / \mathrm{kg}$ (E. coli strain 0111:B4, Sigma-Aldrich, St Louis, MO, USA) as described elsewhere. ${ }^{33}$ Rats ( $n=4-6$ per group) were killed $48 \mathrm{~h}$ after treatment, including a group of naïve animals (experiments were approved by the Animal Care Committee). Lungs were fixed in 10\% formalin and embedded in paraffin.

\section{HBEC Primary Culture}

Normal HBEC (donor 2F1688; Lonza Group, Basel, Switzerland) were cultured using a modified method. ${ }^{34}$ In brief, passage $0(\mathrm{P} 0)$ cells were expanded, and then $\mathrm{P} 2$ cells were seeded in T-162 flasks and grown in $50 \mathrm{ml}$ of BEBM Basal Medium (Lonza) plus BEGM SingleQuots supplements and growth factors (Lonza). Media was changed every $48 \mathrm{~h}$ until cells were $90 \%$ confluent. Then, HBEC were seeded on 12well inserts $\left(8.25 \times 10^{4}\right.$ cells per insert $)$ of transwell-permeable supports (Corning, Corning, NY, USA) adding $1 \mathrm{ml}$ of differentiation media prepared by mixing $250 \mathrm{ml}$ of BEBM media, $250 \mathrm{ml}$ of Dulbecco's modified Eagle medium (Invitrogen, Paisley, UK), BEGM SingleQuots using same supplements as above, but without triiodothyronine, retinoic acid or GA-1000, and supplemented with all trans-retinoic acid (Sigma-Aldrich) at a concentration of $50 \mathrm{nM}$ plus gentamycin $50 \mu \mathrm{g} / \mathrm{ml}$ (Invitrogen). HBEC were maintained submerged in differentiation media for 7 days and then exposed for further 14 days to an air-liquid interface adding into their basolateral chambers $1 \mathrm{ml}$ of MDM supernatant. Controls were performed with differentiation media, LPS (E. coli strain 055:B5, Sigma-Aldrich) $1.67 \mu \mathrm{g} / \mathrm{ml}$, and recombinant human (rh) IL-13 $1 \mathrm{ng} / \mathrm{ml}$ (Peprotech, Rocky Hill, NJ, USA) to induce MUC5AC. ${ }^{10,13}$ Additionally, HBEC were exposed to a combination of rh cytokines (R\&D Systems, Minneapolis, MN, USA) prepared in differentiation media at concentrations equivalent to mean values from MDM supernatant (Figure 4b). Controls included also rh neuregulin-1 $\beta 150 \mathrm{nM} .^{10}$ Each condition was assayed in four to six replicas. At all stages of culture, cells were maintained at $37^{\circ} \mathrm{C}$ in $5 \% \mathrm{CO}_{2}$ in an air incubator. At the end of day 14 , HBEC were fixed in $10 \%$ buffered formalin and embedded in paraffin-blocks as reported. ${ }^{10}$

\section{Lung Tissues and HBEC Staining}

Lung serial sections ( $n=4-6$ per group) were immunostained in Ventana Discovery XT autostainers, (Illkirch Cedex, France) using the DAB map kit as indicated by the manufacture. Working conditions for primary and secondary $\mathrm{Ab}$ are presented in Table 1. Negative controls were performed with IgG isotype control and by omitting primary Ab. Double immunostaining started with mild MCC1 antigen retrieval Kit pre-treatment, followed by primary and 
Table 1 Working conditions for immunostainings performed in rat lung tissues

$\begin{array}{ccc}\text { Primary } \mathrm{Ab} \text { or reagent } & \begin{array}{c}\text { Concentration } \\ (\mu \mathrm{g} / \mathrm{ml}) \text { or } \\ \text { dilution }\end{array} & \begin{array}{c}\text { Incubation } \\ \text { (h) }\end{array} \\ \end{array}$

$\begin{array}{lcc}\begin{array}{l}\text { Monoclonal mouse anti-rat CD68 (clone } \\ \text { ED1) IgG } 1 \text {, Serotec }\end{array} & 0.5 & 1 \\ \begin{array}{l}\text { Monoclonal mouse anti-rat CD103 } \\ \text { (clone Ox-62) IgG } 1 \text {, Serotec }\end{array} & 40 & 12 \\ \begin{array}{l}\text { Monoclonal mouse anti-rat Ki-67 (clone } \\ \text { MIB-5) IgG1, Dako }\end{array} & 15 & 12 \\ \text { Monoclonal mouse anti-rat MHC Class II } & 10 & 12 \\ \text { RT1B (clone Ox-6) IgG } 1 \text {, Serotec } & \\ \begin{array}{l}\text { Polyclonal goat anti-human TLR-4 IgG } \\ \text { (AHP1822), Serotec }\end{array} & 5 & 12 \\ \text { Monoclonal mouse anti-human MU- } & 1 & 1 \\ \text { C5AC (clone 45M1) IgG } 1 \text {, Thermo Fisher } & \\ \text { Scientific } \\ \text { Fluorescein UEA-1, Vector Laboratories }\end{array}$

Secondary $\mathrm{Ab}(\mathrm{IHC}$ protocol)

$\begin{array}{cc}\text { Concentration } & \text { Incubation } \\ (\mu \mathrm{g} / \mathrm{ml}) & (\mathrm{min})\end{array}$

Biotinylated rabbit anti-mouse lgG,

2.6

(CD68), Dako

Biotinylated goat anti-mouse $\lg \mathrm{G}_{1}$,

(CD103 and MHC Class II), Southern

Biotech

Biotinylated goat anti-mouse $\lg \mathrm{G}_{1}$,

(MUC5AC), Southern Biotech

Biotinylated rabbit anti-goat IgG, (TLR-4),

8

32

Dako

Biotinylated rabbit anti-mouse lgG, (Ki-

67), Dako

Biotin-SP-conjugated IgG fraction

monoclonal mouse anti-fluorescein,

(UEA-1), Jackson ImmunoResearch

Laboratories

Abbreviations: MHC, major histocompatibility complex; TLR, Toll-like receptors; UEA-1, Ulex Europaeus Agglutinin 1.

secondary $\mathrm{Ab}$ incubations for major histocompatibility complex (MHC) Class II and Ki-67 as described in Table 1, and the DAB map kit. Then, a denaturation step $\left(95^{\circ} \mathrm{C}\right.$ for $8 \mathrm{~min}$ ) was performed, followed by primary and secondary $\mathrm{Ab}$ incubations for CD68 as shown in Table 1, and the Redmap detection kit. Additional single staining and negative controls were performed. Periodic Schiff's acid staining
(PAS) was performed in $0.5 \%$ periodic acid (Sigma-Aldrich) solution for $5 \mathrm{~min}$. Lung tissue analysis was performed in large and small intra-pulmonary airways such as bronchioles and terminal bronchioles, respectively. They had no cartilage and submucosal glands, and bronchioles were considered only when one or more bronchus-associated lymphoid tissues (BALT) were detected as part of their structure. Bronchiolar compartments proximal (next) to the follicleassociated epithelium (FAE) and distal (opposite to the BALT or in the middle point between two BALT) were investigated for the presence of goblet cells and epithelial cell proliferation. We used PAS, MUC5AC, and the lectin Ulex Europaeus Agglutinin I (UEA-1) as mucin markers, which have been extensively used to study airway goblet cells in rat. ${ }^{35,36}$ Immune markers were assessed in inflamed and non-inflamed areas from bronchioles. Goblet cells and proliferating $\mathrm{Ki}-67^{+}$epithelial cells were quantified by counting stained cells per 50 epithelial nuclei in three different fields of $\times 400$ magnification, excluding unspecific reactions and structures lacking a nucleus, whereas CD68 quantification was performed by percentage of stained area per $\times 400$ field due to the patchy pattern of this marker and difficulties identifying single $\mathrm{CD} 68^{+}$cells. A total of 3-4 different bronchioles and terminal bronchioles were analyzed per rat.

As previously published, ${ }^{10,13}$ serial sections from HBEC ( $n=4-6$ per group) were immunostained for MUC5B and MUC5AC in Ventana Discovery XT autostainers, and 6-10 areas/tissue section were selected to perform semiautomated quantification of the immunostaining under the command of previously validated custom-written macros, respectively. In our hands, this method allows detection of MUC5B and MUC5AC in HBEC, and on top of the apical surface of the epithelium as well (unpublished data). We also performed Toll-like receptor (TLR)-4 immunostaining as described in Table 1. To compare results from different experiments, data were normalized by expressing stained area as a ratio versus the corresponding value of media.

\section{MDM and Supernatant}

PBMC from six adult healthy volunteers were isolated using Ficoll-Paque PLUS (GE Healthcare Bio-Sciences AB, Uppsala, Sweden) and negatively selected for CD3, CD7, CD16, CD19, CD56, CD123 and Glycophorin A with a magnetic labeling system and corresponding LS columns (Monocyte Isolation Kit II, Miltenyi Biotec, Auburn CA, USA). PBMC were cultured in six-well plates $\left(6.25 \times 10^{5}\right.$ cells per well $)$ with $2.5 \mathrm{ml}$ of complete RPMI-1640 medium, plus rh M-CSF $100 \mathrm{ng} / \mathrm{ml}$ (R\&D Systems), and fed again with rh M-CSF on day 3. At day 7, MDM were cultured for $24 \mathrm{~h}$ with $2.5 \mathrm{ml}$ per well of differentiation media with $1.67 \mu \mathrm{g} / \mathrm{ml}$ of LPS or without LPS (control). Supernatant were collected, centrifuged to avoid cell debrids, aliquoted, and stored at $-80^{\circ} \mathrm{C}$ until required. MDM were washed twice in PBS, treated with $1 \mathrm{ml}$ per well of accutase dissociation buffer (PAA Laboratories $\mathrm{GmbH}$, Austria) and incubated for $45 \mathrm{~min}$ at $37^{\circ} \mathrm{C}$. Detached cells 
were counted $\left(4.9-6.2 \times 10^{5}\right.$ per $\left.\mathrm{ml}\right)$ and their viability (94.998.0\%) determined by trypan blue on Beckman Coulter Vi-CELL XR (Beckman Coulter, Fullerton, CA, USA). Fluorescence-activated cell sorting (FACS) analysis of MDM was set up by generating seven tubes/donor $\left(10^{5}-10^{6}\right.$ cells per tube). One tube was used as a negative control without $\mathrm{Ab}$, whereas the other six were incubated with APC-conjugated monoclonal mouse anti-human CD14 $\operatorname{IgG}_{2 \mathrm{a}}$ (Serotec), FITCconjugated monoclonal mouse anti-human $\mathrm{CD} 68 \mathrm{IgG}_{1}$ (clone KP1, Dako), APC-conjugated anti-human CD1a IgG (BD Biosciences, San Diego, CA, USA), APC-conjugated mouse $\operatorname{IgG}_{2 \mathrm{a}}$ (Serotec), FITC-conjugated mouse $\operatorname{IgG}_{1}$ (Dako), or APC-conjugated mouse $\mathrm{IgG}_{1}$ (BD Biosciences), respectively. Tubes for CD68 intracellular staining were washed twice with $0.5 \mathrm{ml}$ of FACS buffer prepared with DPBS (Invitrogen), plus $0.1 \% \mathrm{NaN}_{3}$ (Sigma-Aldrich) and $0.2 \%$ Bovine Albumin Fraction V solution (Invitrogen). Cells were fixed, permeabilized using the IntraStain kit (Dako), and treated with $10 \mu \mathrm{l}$ of the corresponding $\mathrm{Ab}$ or isotype control for $15 \mathrm{~min}$ at RT in the dark. Cells were washed twice with FACS buffer, centrifuged, and resuspended in $0.5 \mathrm{ml}$ of FACS buffer for FACS analysis in a FACS CantoTMII flow cytometer (BD Biosciences). Tubes for CD1a and CD14 surface staining were washed twice with FACS buffer. Cells were centrifuged, resuspended in $0.5 \mathrm{ml}$ of FACS buffer, treated with 20 BL of human FcR blocking reagent (Miltenyi Biotec), followed by $10 \mathrm{BL}$ of $\mathrm{Ab}$ for $30 \mathrm{~min}$ at $4{ }^{\circ} \mathrm{C}$ in the dark. Then, cells were washed with $2 \mathrm{ml}$ of FACS buffer, centrifuged, and resuspended in $0.5 \mathrm{ml}$ of FACS buffer for analysis.

\section{Cytokine Assay}

Enzyme-linked immunosorbent assay (ELISA) kits for IL-4, IL-13, IL-17, and TGF- $\beta 1$ (R\&D Systems), or a 96-well MULTI-ARRAY MULTI-SPOT electrochemiluminescence assay (Meso-Scale Discovery, Gaithersburg, MD, USA) for IL-1 $\beta$, IL-6, IL-8, IL-10, GM-CSF, and TNF- $\alpha$ were used to study MDM supernatant. Concentrations were determined in triplicate and interpolated from standard curves run concurrently. The limit of detection for the assays in $\mathrm{pg} / \mathrm{ml}$ was: 2.4 for IL- $1 \beta, 0.1$ for IL-4, 4.0 for IL-6, 6.0 for IL-8, 2.4 for IL-10, 93.0 for IL-13, 15.0 for IL-17, 1.0 for GM-CSF, 4.6 for TGF- $\beta 1$, and 6.0 for TNF- $\alpha$.

\section{Statistical Analysis}

We performed Student's $t$-test or one-way ANOVA and Tukey post hoc test considering significant $P<0.05$. Data are presented as mean \pm s.d.

\section{RESULTS}

\section{Phenotype of Bronchioles and Terminal Bronchioles}

Number of UEA-1, MUC5AC, and PAS-positive goblet cells in the rat lung was increased $(P<0.05)$ in bronchiolar FAE proximal and distal areas-but not in terminal bronchiolesfrom LPS-treated animals (Figure $1 \mathrm{e}-\mathrm{g}$ and $\mathrm{m}-\mathrm{o}$, and graph 1 from Figure 1) when compared with controls (Figure 1a-c and $\mathrm{i}-\mathrm{k}$ ). Differences between FAE proximal and distal areas were detected only in controls, where increased $(P<0.05)$ number of UEA- ${ }^{+}$cells was observed in FAE proximal areas (graph 1 from Figure 1). In FAE from controls, some UEA- $^{+}$cells have the morphology of M-cells engulfing small mononuclear cells (inset, Figure 1a), similar to the UEA-1 ${ }^{+}$M-cells reported in nasal FAE from rats. ${ }^{37}$ We performed Ki-67 immunostaining to confirm that the high number of bronchiolar goblet cells from LPS-treated rats was hyperplasia related to abnormal epithelial cell proliferation rather than metaplasia. Results showed augmented $\mathrm{Ki}-67^{+}$ epithelial cells $(P<0.05)$ in terminal bronchioles from LPStreated rats (Figure 1p and graph 2 from Figure 1) compared with the bronchiolar epithelium from controls and LPStreated rats (Figure $1 \mathrm{~d}$ and $\mathrm{h}$, respectively), as well as to terminal bronchioles from controls (Figure 1l). Additionally, a monocytic inflammatory infiltrate in bronchioles with GCH was characterized by Ki-67 nuclear pattern (inset Figure 1h) and a predominant CD68 immunophenotype (Figure 2a-c). CD68 was mainly present in inflamed bronchioles from LPS-treated rats when compared with non-inflamed bronchioles from LPS-treated animals and controls (Figure 2d). Scant $\mathrm{CD}^{+} 8^{+}$macrophages co-localized with Ki-67 (Figure 2e), indicating that proliferation occurred mainly in monocytes rather than in $\mathrm{CD}^{+} 8^{+}$macrophages. In inflamed bronchioles, CD68 had similar distribution and morphology than the antigen-presenting cell marker MHC Class II (Figure 3f). In fact, some co-localization between CD68 and MHC Class II was demonstrated (Figure 2e), suggesting an antigenpresenting role for these cells. We investigated the presence of other antigen-presenting cells like dendritic cells (DC) by performing CD103 immunostaining (Figure $3 a-c$ ). We

Figure $1 \mathrm{Immunohistochemistry} \mathrm{and} \mathrm{quantification} \mathrm{from} \mathrm{immunostaining} \mathrm{of} \mathrm{airway} \mathrm{epithelial} \mathrm{cell} \mathrm{markers} \mathrm{in} \mathrm{intra-pulmonary} \mathrm{bronchioles} \mathrm{and} \mathrm{terminal}$ bronchioles from rats treated with intra-tracheal administration of PBS (controls, $n=4)$ or LPS $(n=6)$. Representative microphotographs (magnifications $\times 200$ ) showing mucin marker staining (Ulex Europaeus Agglutinin I (UEA-1), MUC5AC and Periodic Schiff's acid staining (PAS)) as well as the proliferation marker Ki-67 in bronchiolar compartments (a-h) and terminal bronchioles in tissue samples from controls (a-d and $\mathbf{i}-\mathbf{I})$, and LPS-treated (e-h and $\mathbf{m}-\mathbf{p})$ rats (black arrows highlight specific staining). Inserts showing UEA-1 ${ }^{+}$cell in follicle-associated epithelium (FAE) in panel a and Ki-67 ${ }^{+}$infiltrating monocytes from FAE distal area in panel b, respectively. Graphs show number of epithelial cells stained in FAE proximal and distal areas from bronchioles, as well as terminal bronchioles in lung tissues from controls and LPS-treated rats. Graph 1 shows UEA-1, PAS, and MUC5AC (black, gray, and white bars, respectively), whereas graph 2 results for Ki-67. Values are mean \pm s.d. Graph 1: ${ }^{*} P<0.001$ vs UEA-1, PAS, and MUC5AC from small airways control and LPS groups; ${ }^{\dagger} P<0.05$ vs respective control group in bronchioles; ${ }^{* *} P<0.05$ vs PAS and MUC5AC FAE proximal control groups; ${ }^{\circ} P<0.05$ vs all control and LPS groups in bronchioles. Graph 2: ${ }^{*} P<0.05$ vs all other groups. 


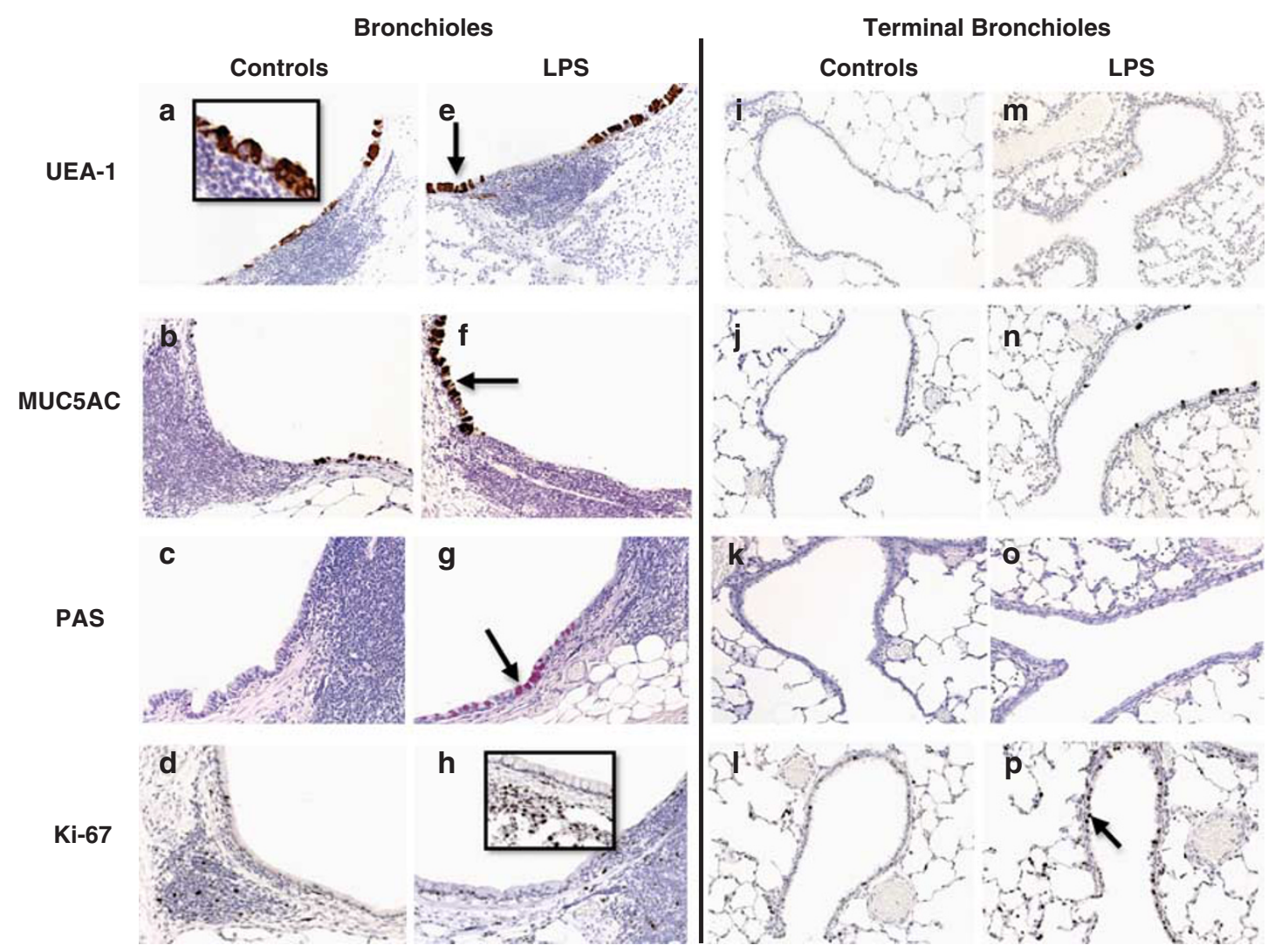

Quantifications

1. Mucin Markers

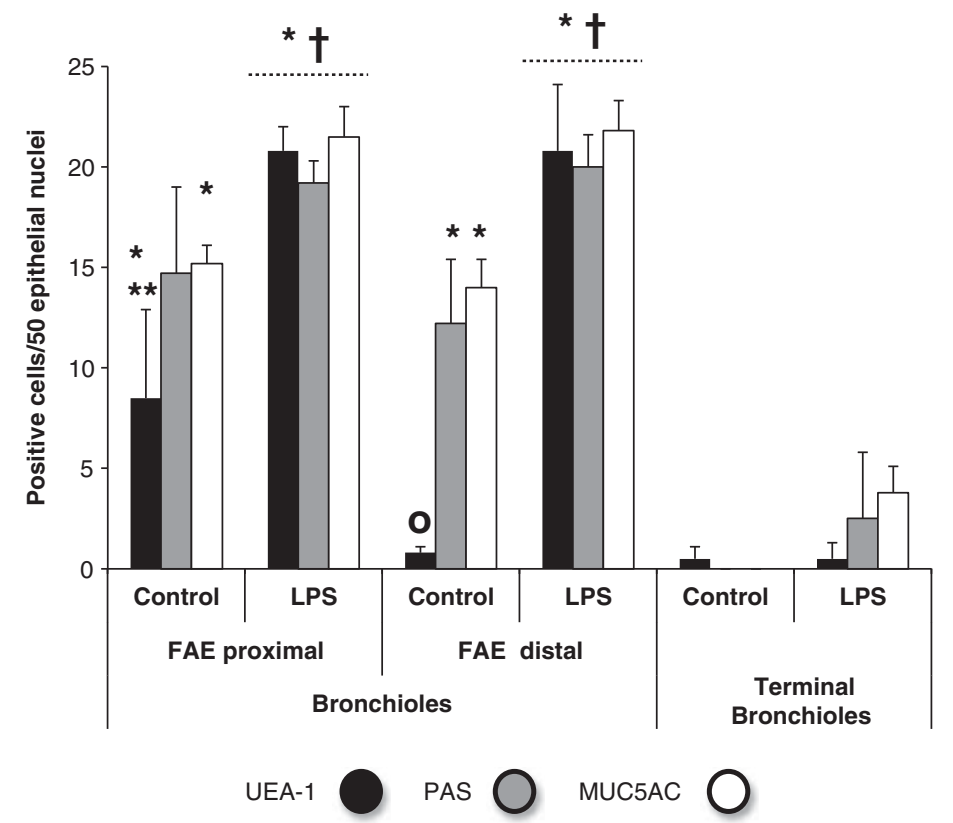

2. Marker of Proliferation Ki-67

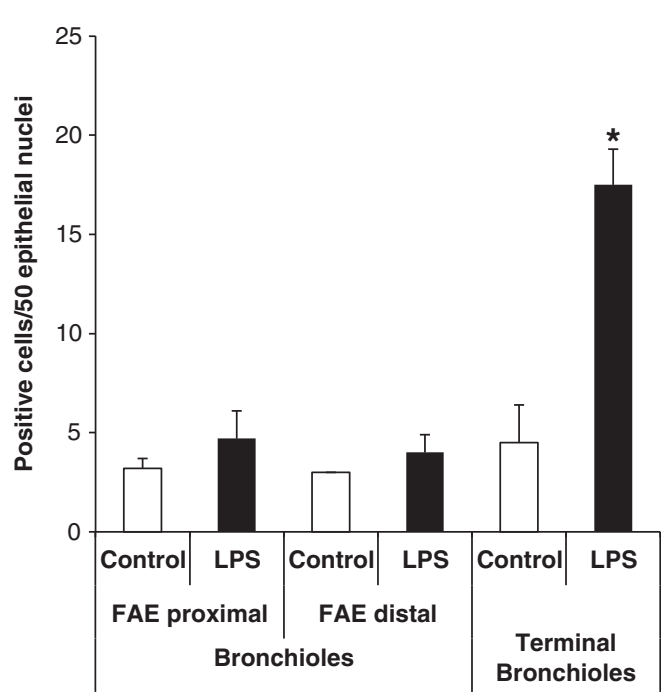

LPS Control $\bigcirc$ 

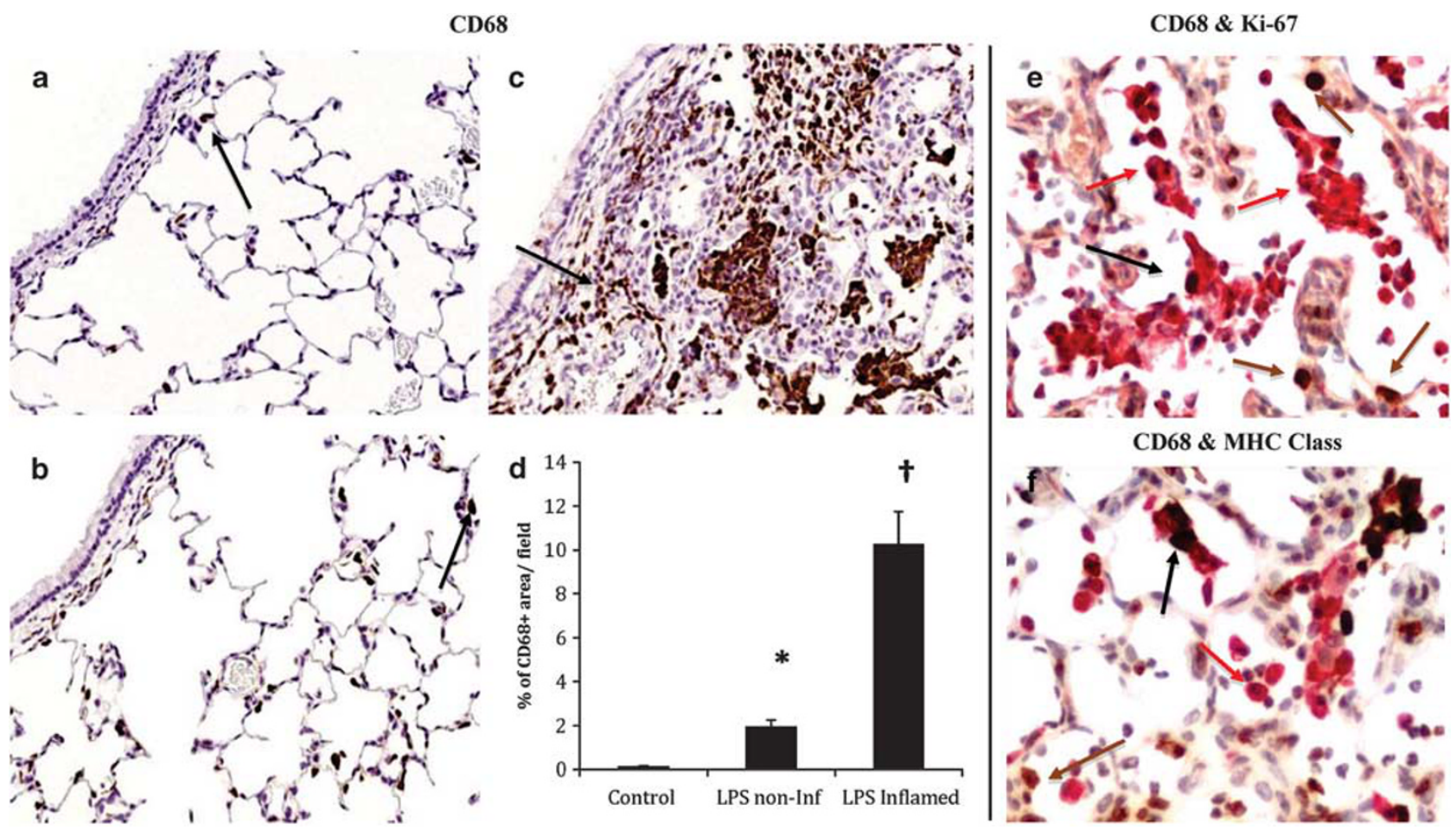

Figure 2 CD68 single and double immunostaining vs Ki-67 or major histocompatibility complex (MHC) Class II in lung tissue samples from rats treated with intra-tracheal administration of PBS (controls, $n=4)$ or LPS $(n=6)$. Representative microphotographs (magnifications $\times 200)$ from single CD68 immunostaining in bronchioles from controls (a), as well as non-inflamed (b) and inflamed (c) areas from LPS-treated rats, respectively (black arrows highlight specific staining, showing interstitial macrophage infiltrate in panel c. Quantification of single CD68 immunostaining (d) performed in controls, as well as non-inflamed and inflamed areas in tissue samples from LPS-treated animals. Values in black bars are mean \pm s.d.; ${ }^{*} P<0.05$ vs control, and ${ }^{\dagger} P<0.001$ vs control and non-inflamed groups. Results of double immunostaining showing Ki- $67^{+} \mathrm{CD}^{-} 8^{-}$monocytes (brown nuclear staining indicated by brown arrows), $\mathrm{Ki}-67^{-} \mathrm{CD}^{+} 8^{+}$macrophages (red cytoplasm; red arrows), and $\mathrm{Ki}-67^{+} \mathrm{CD} 68^{+}$macrophage (brown nuclear staining and red cytoplasm; black arrow) from an inflamed area in (e), whereas MHC Class $\mathrm{II}^{+} \mathrm{CD}^{+} 8^{+}$macrophages (black staining; black arrow), MHC Class $\mathrm{II}^{+} \mathrm{CD}^{-} 8^{-}$(brown staining; brown arrow), and $\mathrm{MHC}$ Class $\mathrm{II}^{-} \mathrm{CD}^{+} 8^{+}$(red staining; red arrow) in inflamed tissue (f). Representative microphotographs (magnification $\times 400$ ).

observed $\mathrm{CD} 103^{+} \mathrm{DC}$ only in bronchioles from control specimens and in non-inflamed bronchioles from LPS-treated rats. The LPS receptor TLR-4 was localized in FAE proximal and distal bronchiolar epithelium from LPS-treated rats only (Figure $3 \mathrm{~g}-\mathrm{i}$ ). However, BALT and FAE from bronchioles shown TLR-4 in the apical compartment of the FAE (inserts, Figure $3 \mathrm{~g}$ and i, respectively), as well as MHC Class II across the BALT and FAE (insets, Figure $3 \mathrm{~d}$ and f, respectively) in tissue samples from controls and LPS-treated rats. Complementary $\mathrm{CD} 3$ immunostaining showed absence of any abnormal lymphocytic infiltrate in bronchioles from LPS-treated rats (data not shown). Lung tissue samples from naïve rats $(n=4)$ stained for all the epithelial and immune markers were similar to controls (data not shown).

\section{Human MDM and Supernatant}

The immunophenotype of MDM incubated with or without LPS was similar, presenting high levels of CD14 and CD68, but low levels of CD1a, respectively (Figure 4a). When their supernatant were analyzed, increased levels of IL- $1 \beta$, IL6, IL-8, IL-10, and TNF- $\alpha$ (all $P<0.05$ ) were found in
MDM samples treated with LPS compared with controls (Figure $4 \mathrm{~b}$ ). Levels of IL-4, IL-13, IL-17, and TGF- $\beta 1$ were undetectable.

\section{Immunophenotype from HBEC Exposed to MDM Supernatant}

HBEC showed unique changes in MUC5B and MUC5AC immunophenotypes when exposed to MDM supernatant (Figure 5). A $100 \%$ increase in MUC5B $(P<0.05)$ was detected in HBEC challenged with supernatant from LPStreated MDM, when compared with media. Minor increases of $80(P<0.05)$ and $60 \%$ in MUC5B were observed in HBEC challenged with LPS and IL-13 compared with media, respectively. On the other hand, $45 \%$ inhibition of MUC5B was detected in HBEC challenged with supernatant from control MDM. MUC5AC immunostaining showed 21\% increase and 36\% inhibition in HBEC challenged with LPS and supernatant from LPS-treated MDM compared with media, respectively. Furthermore, MUC5AC was inhibited by $76 \%$ $(P<0.05)$ in HBEC challenged with supernatant from control MDM compared with media. Conversely, $230 \%$ increase 

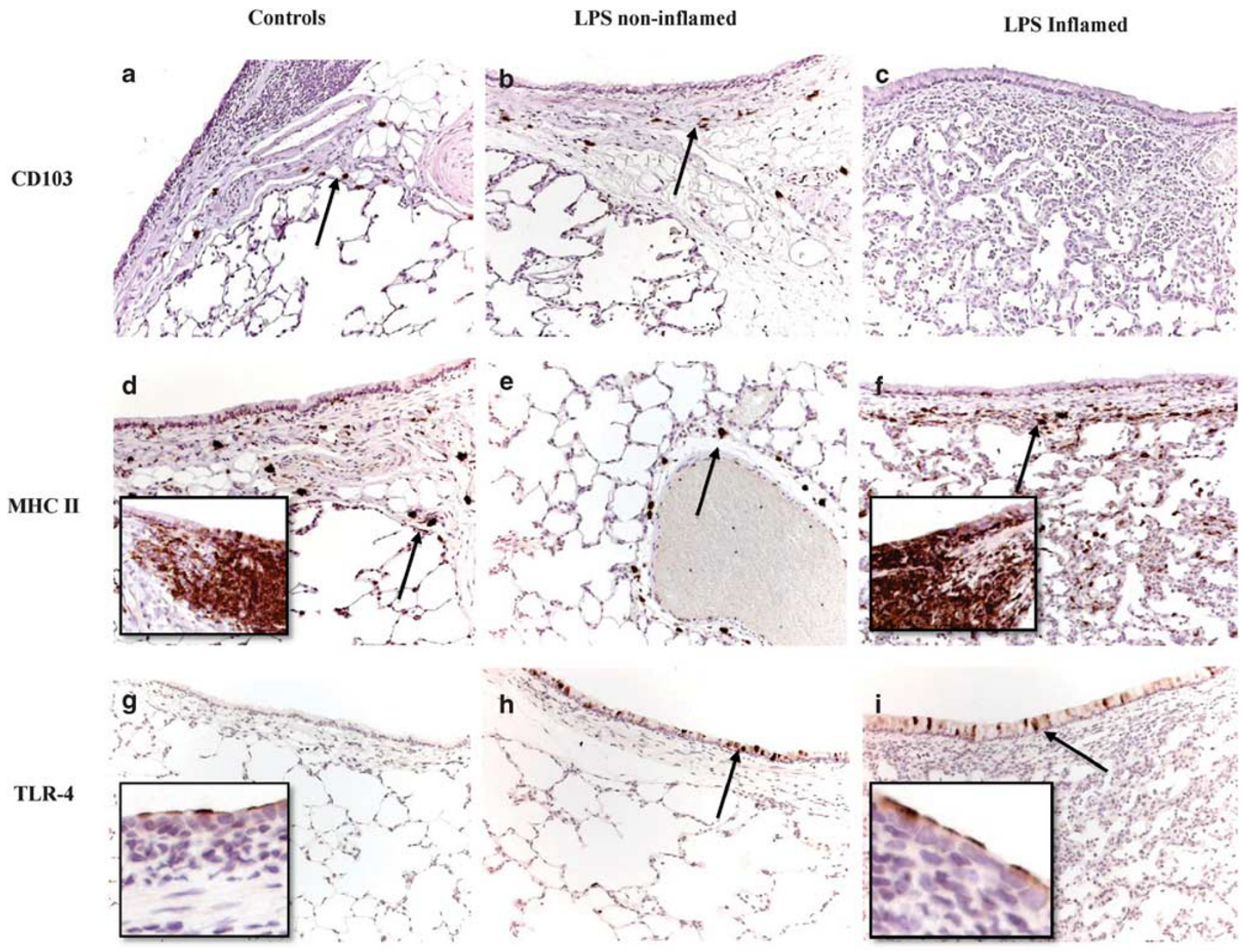

Figure 3 CD103, major histocompatibility complex (MHC) Class II and Toll-like receptor (TLR)-4 immunostaining in bronchioles from rats treated with intra-tracheal administration of PBS (controls, $n=4)$ or LPS $(n=6)$. Representative microphotographs for CD103 immunostaining (a-c), MHC Class II (d-f), and TLR-4 (g-i) are shown (magnifications $\times 200$; black arrows highlight specific staining). Results from controls $(\mathbf{a}, \mathbf{d}, \mathbf{g})$ and tissue samples obtained from LPS-treated animals in non-inflamed $(\mathbf{b}, \mathbf{e}, \mathbf{h})$ and inflamed $(\mathbf{c}, \mathbf{f}, \mathbf{i})$ areas, respectively. Results of MHC Class II (d, $\mathbf{f}$; insets $\times 200)$ and TLR-4 (g, i; insets $\times 400$ ) in follicle-associated epithelium (FAE) and bronchus-associated lymphoid tissue (BALT) present in bronchioles from controls (d, $\mathbf{g}$ ) and LPS-treated rats $(\mathbf{f}, \mathbf{i})$, respectively.

$(P<0.05)$ in MUC5AC immunostaining was detected in HBEC challenged with IL-13. MUC5B and MUC5AC immunostaining were observed inside HBEC, but not on top of their apical poles. Finally, absence of specific TLR-4 immunostaining was observed in HBEC treated with media, media + IL-13, media + LPS, as well as supernatant from LPS-treated MDM or control MDM (not shown data).

\section{Immunophenotype from HBEC Exposed to Cytokines}

We investigated if cytokines at levels present in supernatant from MDM could contribute to induce changes on MUC5B and MUC5AC immunophenotypes (Figure 6). Results show that HBEC challenged with a mix of GM-CSF, IL- $1 \beta$, IL-6, IL-8, IL-10, and TNF- $\alpha$ at concentrations similar to supernatant from LPS-treated MDM (referred as high cytokine levels), as well as TNF- $\alpha$ alone, consistently increased
MUC5B immunophenotype compared with media (Figure 6a and c). The mix of cytokines at high levels (Figure 6a) induced MUC5B in a range of 80 to $220 \%$ (average 130\%) compared with media, which was similar to the effect of TNF- $\alpha$ alone (range 100 to $180 \%$, average $130 \%$ ). GM-CSF, IL-6, and IL- $1 \beta$ induced MUC5B immunophenotype only in one out of three experiments, which was not considered a consistent positive result (Figure 6a). GM-CSF, IL-6, IL-8, IL-10, and TNF- $\alpha$ at concentrations equivalent to supernatant from control MDM (low cytokine levels) did not generate consistent changes of MUC5B in the three experiments performed (Figure $6 \mathrm{~b}$ ). In addition, controls treated with LPS or neuregulin-1 $\beta 1$ induced a consistent increase of MUC5B in the three experiments when compared with media (average 70 and $370 \%$, respectively), whereas IL-13 did not alter MUC5B (Figure 6c). 
a

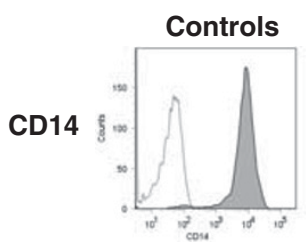

MDM Immunophenotype
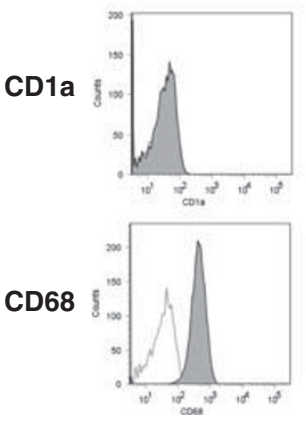

\begin{tabular}{|c|c|}
\hline & $\begin{array}{c}\text { \% of Positive } \\
\text { Cells }\end{array}$ \\
\hline CD14 & $92.9 \pm 7.2$ \\
\hline CD1a & $5.2 \pm 1.2$ \\
\hline CD68 & $99.4 \pm 0.6$ \\
\hline
\end{tabular}
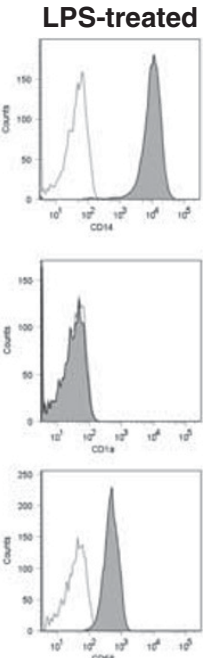

\begin{tabular}{|c|c|}
\hline & $\begin{array}{c}\% \text { of Positive } \\
\text { Cells }\end{array}$ \\
\hline CD14 & $97.4 \pm 2.8$ \\
\hline CD1a & $5.0 \pm 2.3$ \\
\hline CD68 & $99.4 \pm 0.4$ \\
\hline
\end{tabular}

b

MDM Supernatant Cytokines

\begin{tabular}{|c|c|c|}
\hline \multicolumn{1}{c}{ Controls } & LPS-treated \\
\hline IL-1 $\beta$ & $0 \pm 0$ & $17 \pm 10 *$ \\
\hline IL-6 & $5 \pm 9$ & $642 \pm 754 *$ \\
\hline IL-8 & $1,469 \pm 1,599$ & $41,213 \pm 23,664 *$ \\
\hline IL-10 & $87 \pm 22$ & $3,618 \pm 1,113 *$ \\
\hline GM-CSF & $3 \pm 3$ & $18 \pm 16$ \\
\hline TNF- $\alpha$ & $11 \pm 4$ & $585 \pm 504 *$ \\
\hline
\end{tabular}

Figure 4 Fluorescence-activated cell sorting (FACS) immunophenotype (a) and enzyme-linked immunosorbent assay (ELISA) cytokine levels released in supernatant (b) from monocyte-derived macrophages (MDM) obtained from healthy donors $(n=6) 24 \mathrm{~h}$ after LPS or media challenge (control). MDM immunophenotype from one representative donor are shown in panel a. Histograms for CD14 (monocyte marker), CD1a (dendritic cell (DC) marker), and CD68 (macrophage marker) present overlay of fluorescence from isotype-matched Ig of inappropriate specificity (red line) and their respective specific antibody ( $\mathrm{Ab}$; solid green). Summary of the percentage of positive stained cells for the six donors are shown in each table (mean $\pm \mathrm{s} . \mathrm{d}$.). Cytokine levels (pg/ $\mathrm{ml}$ ) from MDM supernatant are shown in panel b. Values are mean \pm s.d.; $P<0.05$ vs control.

HBEC challenged with a mix of cytokines, as well as by TNF- $\alpha$ or IL- $1 \beta$ at high levels, generated a consistent increase of MUC5AC immunophenotype in these three experiments (Figure $6 \mathrm{~d}-\mathrm{f}$ ). Compared with media, the mix of cytokines at high levels induced MUC5AC in a range of 180 to $220 \%$ (average 200\%); TNF- $\alpha$ increased MUC5AC in a range of 90 to $200 \%$ (average $150 \%$ ) and IL- $1 \beta$ induced MUC5AC in a range of 30 to $200 \%$ (average $90 \%$; Figure 6d). GM-CSF, IL-6, IL-8, and IL-10 increased MUC5AC only in one or two experiments, which were not considered consistent and satisfactory outcomes. When HBEC were challenged with low cytokine levels, none of them was able to induce consistent changes of MUC5AC (Figure 6e). Controls such as LPS and neuregulin- $1 \beta 1$ produced consistent increase of MUC5AC in these three experiments compared with media (average 50 and $180 \%$, respectively), whereas IL-13 increased MUC5AC only in two assays (Figure 6f). Finally, we did not detect MUC5B or MUC5AC on top of HBEC apical poles (unpublished pictures).

\section{DISCUSSION}

In our rat model of LPS-induced pulmonary inflammation, a close anatomical association between bronchiolar GCH and macrophage infiltration suggests that macrophages may have a role modulating the airway goblet cell phenotype. When investigating this hypothesis in vitro, we found that supernatant from LPS-treated or control MDM induced unique alterations of MUC5B and MUC5AC immunophenotypes in primary HBEC culture, which were partially reproduced by TNF- $\alpha$ at levels similar to those in supernatant. Moreover, MUC5AC was significantly inhibited by supernatant from control MDM and no tested cytokine could reproduce this effect, indicating that other soluble factor(s) might be involved. Conversely, MUC5B was induced by LPS, but also by supernatant from LPS-treated MDM and TNF- $\alpha$. We propose that this combination of mechanisms could be responsible for $\mathrm{MUC}_{5 \mathrm{~B}}{ }^{+} \mathrm{GCH}$ in pulmonary inflammation.

Episodes of airway infection in CF, COPD, and asthma are frequently associated with mucus hypersecretion and disease exacerbation. ${ }^{1,11}$ These infections can be induced by different types of microbes that provide a wide source of antigenslike LPS from Gram-negative bacteria-able to provoke a quick innate response when recognized by the airway epithelium. ${ }^{38}$ Moreover, inhalation of air contaminated with LPS is associated with airway obstruction and inflammation, ${ }^{39}$ signs present in these respiratory disorders. Single intra-tracheal administration of LPS from $P$. aeroginosa or E. coli in rat and mouse has been extensively used to induce 


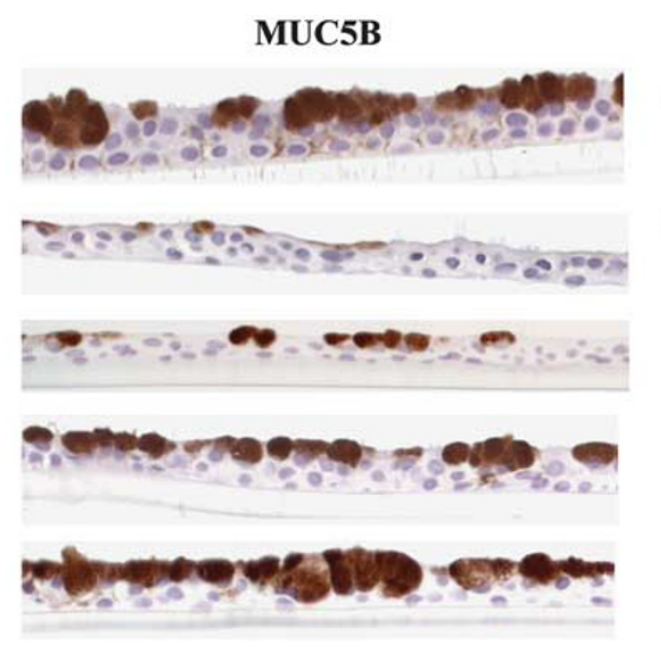

\section{MDM Supernatant \\ + LPS}

MDM Supernatant

No LPS

Media

Media + LPS
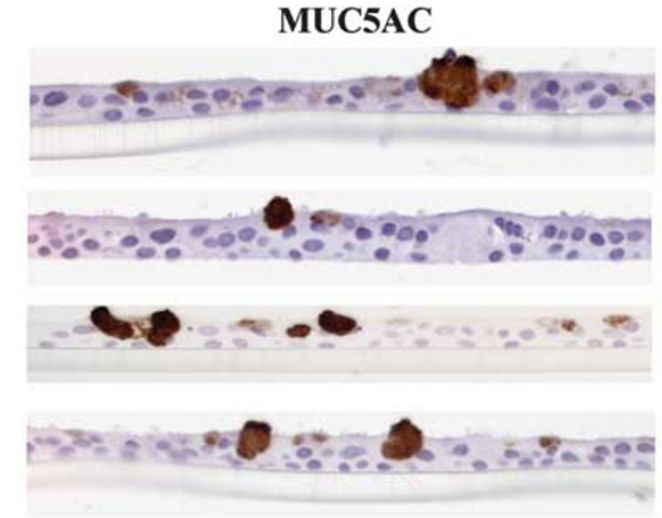

Media + IL-13
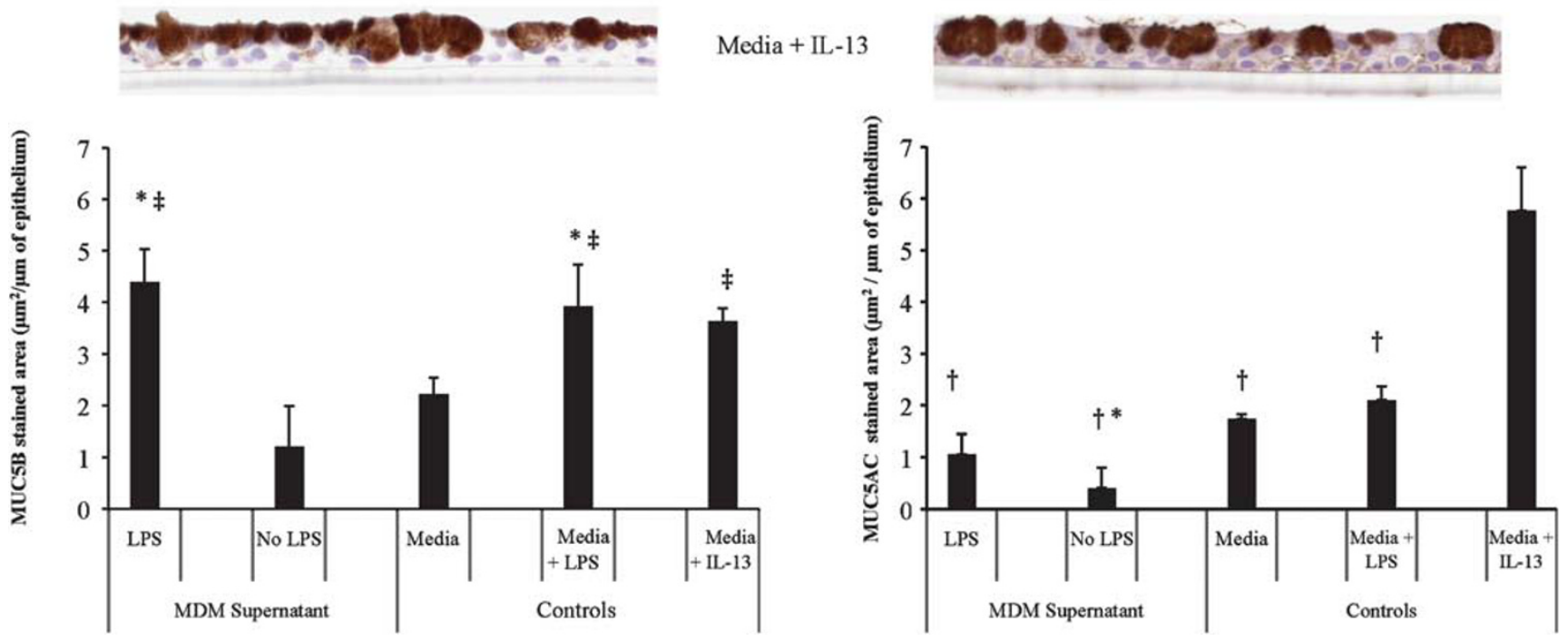

Figure 5 MUC5B and MUC5AC immunostaining and quantifications from immunostaining of human bronchial epithelial cells after 2 weeks of culture in air-liquid interface with basolateral exposition to supernatant obtained from monocyte-derived macrophages (MDM) cultured with or without LPS ( $n=6$ per group). Basolateral stimulation with media alone (Media), Media + LPS $(1.67 \mu \mathrm{g} / \mathrm{ml})$, and Media $+\mathrm{IL}-13$ (1 $\mathrm{ng} / \mathrm{ml})$ were performed as controls. Representative microphotographs for both MUC5B and MUC5AC are shown (magnifications $\times 400$ ). Quantifications for MUC5B and MUC5AC are shown in graphs. Values in black bars are mean \pm s.d.; ${ }^{\ddagger} P<0.05$ vs no LPS; ${ }^{*} P<0.05$ vs Media; and ${ }^{\dagger} P<0.05$ vs Media + IL-13.

mucus production. ${ }^{35,40-42}$ In the present study, rats treated with a single intra-tracheal dose of LPS from E. coli show increased number of goblet cells only in bronchiolar areas proximal and distal to the FAE, but not in terminal bronchioles. We did not investigate if increased number of goblet cells was associated with mucin changes in bronchoalveolar lavage, because airway mucin secretion was out of the scope of this study. Of note, augmented epithelial cell proliferation equivalent to the number of goblet cells in terminal bronchioles confirmed the presence of GCH and not metaplasia, as proposed by others. ${ }^{35} \mathrm{~A}$ differential distribution of goblet cells and proliferative epithelial cells indicate the relevance of studying specific anatomical compartments. It is also important to highlight that mucin induction by a single intra-tracheal administration of LPS in mouse and rat is different, because the upregulation of mucin markers in mouse occurs at day $7,{ }^{42}$ and not $48 \mathrm{~h}$ after treatment like in rats. In addition, LPS challenge for $48 \mathrm{~h}$ in mouse generates a neutrophilic $^{42}$ rather than a mononuclear/monocytic inflammatory response. Differences between mouse and rat towards LPS treatment may be likely related to differences in their mucosal immune systems, such as the presence of BALT, and subsequently FAE in rats, but not in normal mouse. ${ }^{43}$ In fact, TLR-4 and MHC Class II detected in bronchiolar FAE from control rats suggests that LPS uptake may occur in this compartment during the onset of lung inflammation. In parallel, we showed a predominant CD68 macrophage infiltrate in bronchioles, similar to another study, ${ }^{44}$ but no lymphocytic or polymorphonuclear (neutrophilic) response was observed. Although neutrophils have a key role in LPSinduced lung inflammation, ${ }^{39}$ rats challenged with single intra-tracheal administration of LPS present pulmonary neutrophilic infiltration peaking at 6-24 $\mathrm{h}$ with restoration to baseline levels at $48-72 \mathrm{~h}$, whereas a delayed monocytic infiltrate occur at $24-48 \mathrm{~h}$ after treatment, ${ }^{44}$ in agreement with our observations. Unfortunately, this data does not 
a
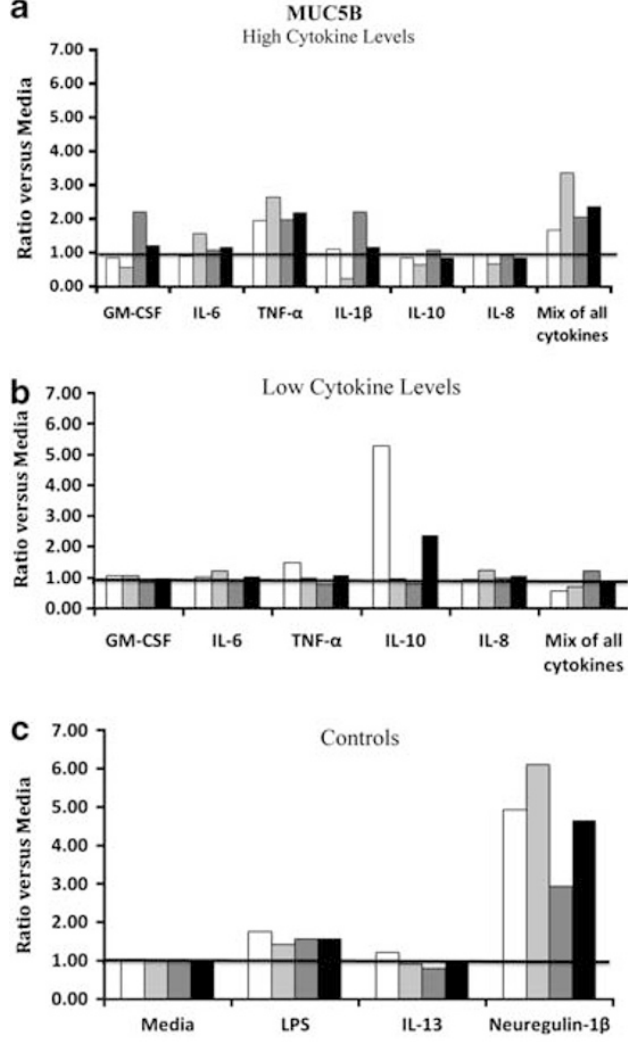

d
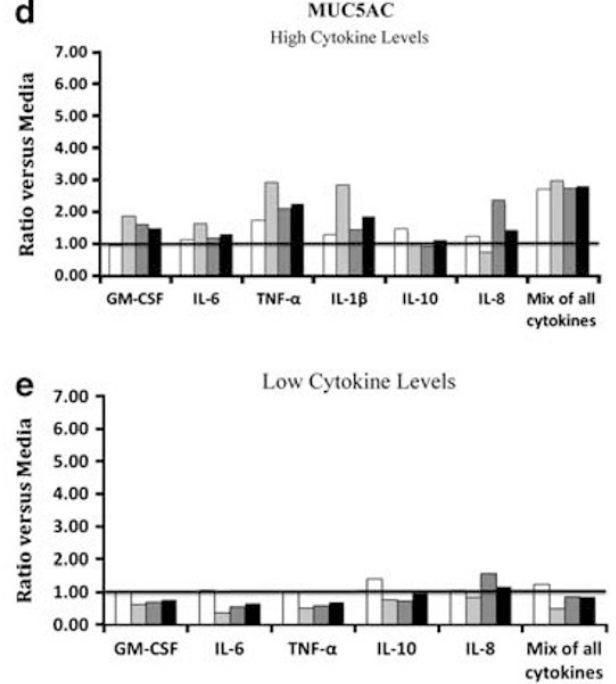

f

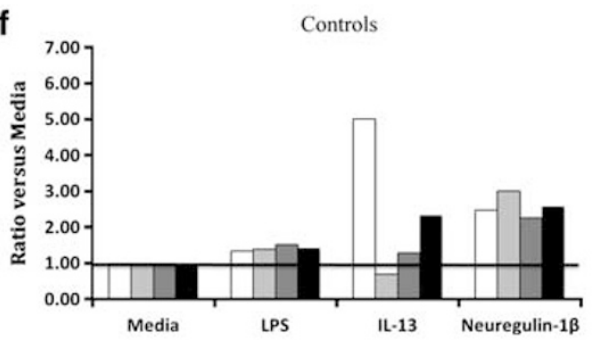

White: Exp 1 Light gray: Exp 2 Dark gray: Exp 3 Black: Average 1-3

Figure 6 MUC5B and MUC5AC quantifications from immunostaining in human bronchial epithelial cells after 2 weeks of culture in air-liquid interface with basolateral exposition to cytokines at high (a and $\mathbf{d}$ ) and low levels (b and $\mathbf{e})$, respectively. Basolateral stimulation with media alone (media), LPS, IL-13, and neuroregulin-1 $\beta 1$ were performed as controls (c and f). Results from three independent experiments (Exp 1, Exp 2, and Exp 3), including their average (Average 1-3), are shown. All conditions were assayed in four to six replicates. Bars represent mean values. Black horizontal line across graphs is showing basal levels for media.

clarify whether an early neutrophilic and/or a later monocytic infiltrate induces GCH. However, our association between bronchiolar GCH and abnormal macrophage infiltrate suggests that these two events might be correlated. We investigated other monocytic-derived innate cell types showing that $\mathrm{CD} 103^{+} \mathrm{DC}$ were present in the connective tissue from non-inflamed bronchioles - in accordance with a previous report ${ }^{45}$-but not in inflamed areas. CD103 is a rat-specific DC marker ${ }^{46}$ that may account only for a sub-population of lung DC. MHC Class II immunostaining-capable to detect various types of DC and other antigen-presenting cellsidentified cells in inflamed areas with similar morphology and allocation as CD68, but both markers only partially colocalized. Thus, sub-populations of $\mathrm{MHC}$ Class $\mathrm{II}^{+} \mathrm{CD} 68^{+}$ and MHC Class $\mathrm{II}^{-} \mathrm{CD} 68^{+}$lung macrophages co-exist with MHC Class $\mathrm{II}^{+} \mathrm{CD}^{-} 8^{-}$cells. Additionally, TLR-4 immunostaining showed presence of LPS receptor only in the epithelium, but not in the inflammatory infiltrate, as reported elsewhere. ${ }^{40}$ In conclusion, an association between bronchiole GCH and lung macrophage infiltration induced by exposure to a bacterial antigen was found in rats.
Absence of luminal macrophages in the rat bronchioles suggest that an interaction between them and the apical surface of the airway epithelium may not have a predominant role in our model, although luminal macrophages could have been removed by lung inflation during fixation. Moreover, we did not have bronchoalveolar fluids to examine luminal inflammatory cells as performed in other studies. ${ }^{35,36} \mathrm{An}$ interaction between the apical pole of the airway epithelium and luminal microbial antigens may induce the release of cytokines that trigger GCH via local stimulation or recruitment of additional inflammatory cells. ${ }^{38}$ However, the close vicinity detected in our LPS model between bronchiole goblet cells and interstitial macrophages allow us to hypothesize that-beside any direct cell-cell interaction-soluble factors released by those macrophages may contribute also to modulate via a basolateral pathway the phenotype of bronchiolar goblet cells. Because of structural differences between the rat and human airway mucosal immune systems represented by the absence of BALT in healthy humans, ${ }^{43}$ we decided to further validate our hypothesis in a human-based model rather than in rats stimulating in-vitro primary culture 
of HBEC with supernatants from human MDM challenged with or without LPS.

Macrophages were generated in vitro by differentiating PBMC in the presence of M-CSF and not GM-CSF, ${ }^{18,19}$ as this growth factor may provide a better method to produce interstitial-like macrophages. ${ }^{32}$ In agreement with other studies, ${ }^{18-20}$ MDM challenged with LPS released high levels of pro-inflammatory cytokines such as IL- $1 \beta$, IL-6, IL-8, and TNF- $\alpha$, as well as high levels of the regulatory IL-10, suggesting the presence of a mix of inflammatory and regulatory MDM. Human PBMC are a source of heterogeneous MDM progenitors, ${ }^{18,19}$ which may generate different sub-populations of macrophages with unique functions and cytokine profiles.

Basolateral exposition of HBEC to MDM supernatant, LPS, and cytokines produced remarkable changes in mucin immunophenotypes. Partial inhibition of MUC5AC was induced by supernatant from LPS-treated MDM in contrast to the consistent increase of MUC5AC generated by a mix of pro-inflammatory cytokines, as well as by TNF- $\alpha$ and IL- $1 \beta$ alone, at levels found in MDM supernatant. However, our findings are in agreement with reports showing that IL-1 $\beta^{47-49}$ and TNF- $\alpha{ }^{47}$ induce MUC5AC mRNA expression in HBEC. Additionally, significant inhibition of MUC5AC was observed in HBEC treated with supernatant from control MDM, whereas lack of consistent MUC5AC changes were observed in HBEC challenged with cytokines mimicking supernatant from control MDM. Therefore, our findings suggest that MDM may release other factor(s) able to downregulate MUC5AC, neutralizing the effect induced by high levels of TNF- $\alpha$ and IL- $1 \beta$. This MUC5AC inhibitory effect in HBEC basolaterally stimulated with supernatant from control and LPS-treated macrophages in vitro allow us to speculate that bronchial interstitial macrophages may also release similar MUC5AC inhibitory factor(s) in healthy and diseased tissues. Proof of this concept could lead to novel therapeutic strategies targeting, for example, lung macrophages and/or their released factor(s) to prevent bronchial MUC5AC ${ }^{+} \mathrm{GCH}$ in mild and moderate asthmatic patients. ${ }^{5}$

MUC5B immophenotype was consistently induced in HBEC challenged with supernatant from LPS-treated MDM carrying high levels of TNF- $\alpha$; by a mix of cytokines mimicking LPS-treated MDM supernatant; and by TNF- $\alpha$ alone at levels from LPS-treated MDM supernatant. Therefore, our data demonstrate that TNF- $\alpha$ at levels released by LPStreated MDM was able to induce MUC5B, suggesting that LPS-stimulated macrophages may generate $\mathrm{MUC} \mathrm{B}^{+} \mathrm{GCH}$. TNF- $\alpha$ released by LPS-activated macrophages could be considered as a therapeutic target to prevent mucus formation in chronic pulmonary disorders. This hypothesis is supported by the fact that increased lung macrophages, ${ }^{24,27}$ as well as enhanced MUC5B phenotype, ${ }^{4,6}$ have been shown in COPD and $\mathrm{CF}$ patients, and that high levels of TNF- $\alpha$ were also reported in tissues from individuals with $\mathrm{COPD} .^{50}$ Among COPD patients, a group present chronic bronchitis, and COPD groups with or without chronic bronchitis have similar high amounts of macrophages in bronchial biopsies. ${ }^{51}$ Therefore, we believe that any COPD group with high TNF- $\alpha$ levels in bronchial macrophages could benefit from an anti-TNF- $\alpha$ intervention to inhibit MUC5B ${ }^{+} \mathrm{GCH}$. However, our hypothesis needs to be validated using HBEC from patients, as bronchus cells from a healthy donor can be genetically and functionally different to diseased airway epithelial cells. ${ }^{52}$ Of note, LPS was able to consistently induce mainly MUC5B in HBEC, similar to the effect reported in the goblet cell line HT29-MTX. ${ }^{53}$ Absence of TLR-4 in HBEC challenged with media, LPS, IL-13, and supernatant from control or LPStreated MDM suggests that HBEC may have responded to LPS via a TLR-4-independent pathway like other epithelial cells do. ${ }^{54}$ Moreover, a basolateral HBEC response to LPS could be a relevant defensive mechanism towards infiltrating pathogens, increasing airway mucus to prevent additional microbial invasion. HBEC stimulated with supernatant from control MDM result in a partial inhibition of MUC5B, and none of the cytokines tested could reproduce this effect. Finally, inconsistent changes in mucin phenotypes produced by cytokines like GM-CSF, IL-6, IL-8, IL-10, and IL-13 may reflect the heterogeneous nature of HBEC, which are a mix of epithelial cells from airways and accessory glands. In fact, these primary cells have not been selected or purified according to any specific phenotype.

In summary, a close anatomical association between bronchiolar GCH and interstitial macrophage infiltrate in a rat model of inflammation driven by LPS suggest that macrophages could induce mucin changes. Using primary HBEC culture, supernatants from control and LPS-treated MDM inhibited MUC5AC immunophenotype, whereas supernatant from LPS-treated MDM enhanced MUC5B. Moreover, TNF- $\alpha$ at levels from LPS-treated MDM induced MUC5B. These findings demonstrate that macrophages generate changes in MUC5AC and MUC5B immunophenotypes.

\section{ACKNOWLEDGEMENTS}

We thank the Biomarkers Unit, Respiratory Disease Area, Novartis Institute of Biomedical Research, Horsham, UK, in particular to Monika Fraczek and Clemence Lebret for their technical assistance with the in vitro work, to

Peter Jones and Farheen Raza for their assistance with

immunohistochemistry, and Paul Whittaker for discussion.

\section{DISCLOSURE/CONFLICT OF INTEREST}

The authors declare no conflict of interest.

1. Thornton DJ, Rousseau K, McGuckin MA. Structure and function of the polymeric mucins in airways mucus. Annu Rev Physiol 2008;70: 459-486.

2. Randell $\mathrm{SH}$, Boucher $\mathrm{RC}$. Effective mucus clearance is essential for respiratory health. Am J Resp Cell Mol Biol 2006;35:20-28.

3. Rose MC, Voynow JA. Respiratory tract mucin genes and glycoproteins in health and disease. Physiol Rev 2006;86:245-278.

4. Burgel PR, Montani D, Danel C, et al. A morphometric study of mucins and small airway plugging in cystic fibrosis. Thorax 2007;62:153-161.

5. Ordoñez $\mathrm{CL}$, Khashayar $\mathrm{R}$, Wong $\mathrm{HH}$, et al. Mild and moderate asthma is associated with airway goblet cell hyperplasia and abnormalities in mucin gene expression. Am J Respir Crit Care Med 2001;163:517-523. 
6. Kirkham S, Kolsum U, Rousseau $\mathrm{K}$, et al. MUC5B is the major mucin in the gel phase of sputum in chronic obstructive pulmonary disease. Am J Respir Crit Care Med 2008;178:1033-1039.

7. Koo JS, Yoon JH, Gray T, et al. Restoration of the mucus phenotype by retinoic acid in retinoid-deficient human bronchial cell cultures: changes in mucin gene expression. Am J Resp Cell Mol Biol 1999;20:43-52.

8. Gray T, Koo J-S, Nettesheim P. Regulation of mucous differentiation and mucin gene expression in the tracheobronchial epithelium. Toxicology 2001;160:35-46.

9. Chen $\mathrm{Y}$, Thai $\mathrm{P}$, Zhao $\mathrm{Y}-\mathrm{H}$, et al. Stimulation of airway mucin gene expression by interleukin (IL)-17 through IL-6 paracrine/autocrine loop. J Biol Chem 2003;278:17036-17043.

10. Kettle R, Simmons J, Schindler F, et al. Regulation of neuregulin 1ß1induced MUC5AC and MUC5B expression in human airway epithelium. Am J Respir Cell Mol Biol 2010;42:472-481.

11. Voynow JA, Gendler SJ, Rose MC. Regulation of mucin genes in chronic inflammatory airway diseases. Am J Respir Cell Mol Biol 2006;34:661-665.

12. Kuperman DA, Huang $X$, Koth LL, et al. Direct effects of interleukin-13 on epithelial cells cause airway hyperreactivity and mucin overproduction in asthma. Nature Med 2002;8:885-889.

13. Atherton $\mathrm{HC}$, Jones $\mathrm{G}$, Danahay H. IL-13-induced changes in the goblet cell density of human bronchial epithelial cell cultures: MAP kinase and phosphatidylinositol 3-kinase regulation. Am J Physiol Lung Cell Mol Physiol 2003;285:L730-L739.

14. Zhao J, Maskrey B, Balzar S, et al. Interleukin-13-induced MUC5AC is regulated by 15-lipoxygenase 1 pathway in human bronchial epithelial cells. Am J Respir Crit Care Med 2009;179:782-790.

15. Tanabe T, Kanoh S, Tsushima K, et al. Clarithromycin inhibits interleukin-13-induced goblet cell hyperplasia in human airway cells. Am J Respir Cell Mol Biol 2011;45:1075-1083.

16. Wills-Karp M, Luyimbazi J, Xu X, et al. Interleukin-13: central mediator of allergic asthma. Science 1998;282:2258-2261.

17. Casalino-Matsuda SM, Monzon ME, Day AJ, et al. Hyaluronan fragments/ CD44 mediate oxidative stress-induced MUC5B up-regulation in airway epithelium. Am J Resp Cell Mol Biol 2009;40:277-285.

18. Mosser MD, Edwards JP. Exploring the full expectrum of macrophage activation. Nat Rev Immunol 2008;8:958-969.

19. Gonzalez-Mejia ME, Doseff Al. Regulation of monocyte and macrophage cell fate. Front Biosci 2009;14:2413-2431.

20. Lohmann-Matthes ML, Steinmuller C, Franke-Ullmann G. Pulmonary macrophages. Eur Respir J 1994;7:1678-1689.

21. Staub NC. Pulmonary intravascular macrophages. Ann Rev Physiol 1994;56:47-67.

22. Prokhorova S, Lavnikova N, Laskin D. Functional characterization of interstitial macrophages and subpopulations of alveolar macrophages from rat lung. J Leukoc Biol 1994;55:141-146.

23. Franke-Ullmann G, Pfortner C, Walter $P$, et al. Characterization of murine lung interstitial macrophages in comparison with alveolar macrophages in vitro. J Immunol 1996;157:3097-3104.

24. Hubeau C, Puchelle E, Gaillard D. Distinct patterns of immune cell populations in the lung of human fetuses with cystic fibrosis. J Allergy Clin Immunol 2001;108:524-529.

25. Wenzel SE, Schwartz LB, Langmack EL, et al. Evidence that severe asthma can be divided pathologically into two inflammatory subtypes with distinct physiologic and clinical characteristics. Am J Respir Crit Care Med 1999;160:1001-1008.

26. Barbato A, Turato G, Baraldo S, et al. Airway inflammation in childhood asthma. Am J Respir Crit Care Med 2003;168:798-803.

27. Di Stefano A, Capelli A, Lusuardi M, et al. Severity of airflow limitation is associated with severity of airway inflammation in smokers. Am J Respir Crit Care Med 1998:158:1277-1285.

28. Holgate ST. Pathogenesis of asthma. Clin Exp Allergy 2008;38:872-897.

29. Brennan S. Innate immune activation and cystic fibrosis. Pediatr Respir Rev 2008;9:271-279.

30. Sethi S, Mallia P, Johnston SL. New paradigms in the pathogenesis of chronic obstructive pulmonary disease II. Proc Am Thorac Soc 2009;6:532-534.

31. Becker S, Warren MK, Haskill S. Colony-stimulating factor-induced monocyte survival and differentiation into macrophages in serum-free cultures. J Immunol 1987;139:3703-3709.

32. Akagawa KS, Komuro I, Kanazawa H, et al. Functional heterogeneity of colony-stimulating factor-induced human monocyte-derived macrophages. Respirology 2006;11:S32-S36.
33. Beckmann N, Tigani B, Sugar $R$, et al. Noninvasive detection of endotoxin-induced mucus hypersecretion in rat lung by MRI. Am J Physiol Lung Cell Mol Physiol 2002;283:L22-L30.

34. Danahay $H$, Atherton $H$, Jones $G$, et al. Interleukin-13 induces a hypersecretory ion transport phenotype in human bronchial epithelial cells. Am J Physiol Lung Cell Mol Physiol 2002;282:L226-L236.

35. Kim JH, Lee SY, Bak SM, et al. Effects of matrix metalloproteinase inhibitor on LPS-induced goblet cell metaplasia. Am J Physiol Lung Cell Mol Physiol 2004;287:L127-L133.

36. Hardaker EL, Freeman MS, Dale N, et al. Exposing rodents to a combination of tobacco smoke and lipopolysaccharide results in an exaggerated inflammatory response in the lung. $\mathrm{Br} J$ Pharmacol 2010;160:1985-1996.

37. Takata S, Ohtani O, Watanabe Y. Lectin binding patterns in rat nasalassociated lymphoid tissue (NALT) and the influence of various types of lectin on particle uptake in NALT. Arch Histol Cytol 2000;63:305-312.

38. Kato A, Schleimer RP. Beyond inflammation: airway epithelial cells are at the interface of innate and adaptive immunity. Curr Opin Immunol 2007;19:711-720.

39. Savov JD, Gavett SH, Brass DM, et al. Neutrophils play a critical role in development of LPS-induced airway disease. Am J Physiol Lung Cell Mol Physiol 2002;283:L952-L962.

40. Chen L, Wang T, Zhang JY, et al. Toll-like receptor 4 relates to lipopolysaccharide-induced mucus hypersecretion in rat airway. Arch Med Res 2009:40:10-17.

41. Chapman RW, Minnicozzi M, Celly CS, et al. A novel orally active CXCR1/2 receptor antagonist, Sch527123, inhibits neutrophil recruitment, mucus production, and goblet cell hyperplasia in animal models of pulmonary inflammation. J Pharmacol Exp Ther 2007;322:486-493.

42. Yanagihara K, Seki M, Cheng PW. Lipopolysaccharide induces mucus cell metaplasia in mouse lung. Am J Respir Cell Mol Biol 2001;24:66-73.

43. Randall TD. Bronchus-associated lymphoid tissue (BALT) structure and function. Adv Immunol 2010;107:187-241.

44. Ulich TR, Watson LR, Yin $\mathrm{S}$, et al. The intratracheal administration of endotoxin and cytokines. I. Characterization of LPS-induced IL-1 and TNF mRNA expression and the LPS-, IL-1-, and TNF-induced inflammatory infiltrate. Am J Pathol 1991;138:1485-1496.

45. Holt PG, Schon-Hegrad MA, Oliver J. MHC class II antigen-bearing dendritic cells in pulmonary tissues of the rat. J Exp Med 1988; 167:262-274

46. Brenan M, Puklavec M. The MRC OX-62 antigen: a useful marker in the purification of rat veiled cells with the biochemical properties of an integrin. J Exp Med 1992;175:1457-1465.

47. Koo JS, Kim YD, Jetten AM, et al. Overexpression of mucin genes induced by interleukin-1 beta, tumor necrosis factor-alpha, lipopolysaccharide, and neutrophil elastase is inhibited by a retinoic acid receptor alpha antagonist. Exp Lung Res 2002;28:315-332.

48. Gray $T$, Coakley R, Hirsh A, et al. Regulation of MUC5AC mucin secretion and airway surface liquid metabolism by IL-1beta in human bronchial epithelia. Am J Physiol Lung Cell Mol Physiol 2004;286: 320-330.

49. Fujisawa $T$, Velichko $S$, Thai $P$, et al. Regulation of airway MUC5AC expression by IL-1 $\beta$ and IL-17A: the NF- $\kappa$ B paradigm. J Immunol 2009;183:6236-6243.

50. Sapey E, Wood AM, Ahmad A, et al. TNF $\alpha$ rs361525 polymorphism is associated with increased local production and downstream inflammation in COPD. Am J Respir Crit Care Med 2010;182:192-199.

51. Snoeck-Stroband JB, Lapperre TS, Gosman MM, et al. Chronic bronchitis sub-phenotype within COPD: inflammation in sputum and biopsies. Eur Respir J 2008;31:70-77.

52. Johnson LG, Boyles SE, Wilson J, et al. Normalization of raised sodium absorption and raised calcium-mediated chloride secretion by adenovirus-mediated expression of cystic fibrosis transmembrane conductance regulator in primary human cystic fibrosis airway epithelial cells. J Clin Invest 1995;95:1377-1382.

53. Smirnova MG, Guo L, Birchall JP, et al. LPS up-regulates mucin and cytokine mRNA expression and stimulates mucin and cytokine secretion in goblet cells. Cell Immunol 2003;221:42-49.

54. Bhattacharyya S, Dudeja PK, Tobacman JK. Lipopolysaccharide activates NF-kappaB by TLR4-Bcl10-dependent and independent pathways in colonic epithelial cells. Am J Physiol Gastrointest Liver Physiol 2008;295:G784-G790. 